\title{
BMJ Open How does morphology impact on diastolic function in hypertrophic cardiomyopathy? A single centre experience
}

\author{
Gherardo Finocchiaro, ${ }^{1}$ Francois Haddad, ${ }^{1,4}$ Aleksandra Pavlovic, ${ }^{1}$ \\ Emma Magavern, ${ }^{1}$ Gianfranco Sinagra, ${ }^{2}$ Joshua W Knowles, ${ }^{1,4}$ Jonathan Myers, ${ }^{3}$ \\ Euan A Ashley ${ }^{1,4}$
}

To cite: Finocchiaro G, Haddad F, Pavlovic A, et al. How does morphology impact on diastolic function in hypertrophic

cardiomyopathy? A single centre experience. BMJ Open 2014:4:e004814.

doi:10.1136/bmjopen-2014004814

- Prepublication history for this paper is available online. To view these files please visit the journal online (http://dx.doi.org/10.1136/ bmjopen-2014-004814).

Received 8 January 2014 Revised 6 May 2014

Accepted 9 May 2014 CrossMark

${ }^{1}$ Division of Cardiovascular Medicine, Department of Medicine, Stanford University School of Medicine, Stanford, California, USA

${ }^{2}$ Cardiovascular Department, Ospedali Riuniti and University of Trieste, Trieste, Italy

${ }^{3}$ Veterans Affairs Palo Alto Health Care System, Palo Alto, California, USA

${ }^{4}$ Stanford Cardiovascular Institute, Stanford

Cardiovascular Medicine, Stanford, California, USA

Correspondence to Dr Gherardo Finocchiaro; gherardobis@yahoo.it

\section{ABSTRACT}

Objectives: It is unclear if morphology impacts on diastole in hypertrophic cardiomyopathy (HCM). We sought to determine the relationship between various parameters of diastolic function and morphology in a large HCM cohort.

Setting: Tertiary referral centre from Stanford, California, USA.

Partecipants: 383 patients with HCM and normal systolic function between 1999 and 2011. A group of 100 prospectively recruited age-matched and sexmatched healthy participants were used as controls.

Primary and secondary outcome measures:

Echocardiograms were assessed by two blinded boardcertified cardiologists. HCM morphology was classified as described in the literature (reverse, sigmoid, symmetric, apical and undefined).

Results: Reverse curvature morphology was most commonly observed (218 (57\%). Lateral mitral annular $E^{\prime}<12 \mathrm{~cm} / \mathrm{s}$ was present in $86 \%$ of reverse, $88 \%$ of sigmoid, $79 \%$ of symmetric, $86 \%$ of apical and $81 \%$ of undefined morphology, $p=0.65$. $E / E^{\prime}$ was similarly elevated (E/E': $12.3 \pm 7.9$ in reverse curvature, $12.1 \pm 6.1$ in sigmoid, $12.7 \pm 9.5$ in symmetric, $9.4 \pm 4.0$ in apical, $12.7 \pm 7.9$ in undefined morphology, $p=0.71$ ) and indexed left atrial volume (LAVi) $>40 \mathrm{~mL} / \mathrm{m}^{2}$ was present in $47 \%$ in reverse curvature, $33 \%$ in sigmoid, $32 \%$ in symmetric, $37 \%$ in apical and $32 \%$ in undefined, $p=0.09$. Each morphology showed altered parameters of diastolic function when compared with the control population. Left ventricular (LV) obstruction was independently associated with all three diastolic parameters considered, in particular with LAVi>40 mL/ $\mathrm{m}^{2}$ (OR 2.04 (95\% Cl 1.23 to 3.39), $\left.\mathrm{p}=0.005\right), E^{\prime} \mathrm{E}^{\prime}>15$ (OR $4.66(95 \% \mathrm{Cl} 2.51$ to 8.64), $p<0.001)$ and $E^{\prime}<8$ (OR 2.55 (95\% Cl 1.42 to 4.53$), p=0.001)$. Other correlates of diastolic dysfunction were age, LV wall thickness and moderate-to-severe mitral regurgitation.

Conclusions: In HCM, diastolic dysfunction is present to similar degrees independently from the morphological pattern. The main correlates of diastolic dysfunction are LV obstruction, age, degree of hypertrophy and degree of mitral regurgitation.
Strengths and limitations of this study

- Large population considered, with complete echocardiographic assessment and a subgroup with genotype characterisation.

- First study to consider diastology and relation with morphology.

- Thorough analysis of what are the major determinants of diastolic dysfunction in hypertrophic cardiomyopathy.

- Patients not studied with cardiac MR.

- Diastole not studied with pulmonary flow in all patients.

\section{INTRODUCTION}

Hypertrophic cardiomyopathy (HCM) is an inherited cardiomyopathy characterised by hypertrophy, fibrosis and fibre disarray. ${ }^{1}$

One of the main pathophysiological features of HCM is diastolic dysfunction. The presence of subtle changes in left ventricular (LV) filling may even identify patients with preclinical disease. ${ }^{2}$ Interventions such as medical therapy, alcohol septal ablation and surgical myectomy improve symptoms by reducing the $\mathrm{LV}$ outflow tract gradients. ${ }^{3}$

Various morphological categories have traditionally been used to describe the appearance of the hypertrophic LV and these include: reverse curvature, sigmoid curvature, symmetric (sometimes referred to as concentric) and apical.

The question of whether different morphological subtypes of HCM (which often portend different therapies) are associated with different degrees of diastolic dysfunction is unknown. We hypothesise that the degree of diastolic dysfunction is poorly related to morphology and that it is present despite the presence of a different 
distribution of hypertrophy. Besides, we sought to analyse the echocardiographic correlates of diastolic dysfunction.

\section{METHODS}

\section{Study population}

From January 1999 to November 2011, 410 adult (age 18-91 years) consecutive patients diagnosed with HCM were enrolled in the Stanford Inherited Cardiomyopathy Registry. For the present study, we retrospectively selected and analysed patients with HCM with preserved LV systolic function ( $\mathrm{n}=383$, LV ejection fraction (LVEF) $>55 \%)$. Twenty-seven patients with LV systolic dysfunction and/or with permanent atrial fibrillation at enrolment were excluded from the study.

The diagnosis of HCM was based on the presence of significant LV hypertrophy (end-diastolic wall thickness $\geq 15 \mathrm{~mm}$ at M-mode or two-dimensional (2D) echocardiography) in the absence of other aetiologies (according to international criteria) or wall thickness between 13 and $15 \mathrm{~mm}$, in the presence of an abnormal ECG or familial history of inherited cardiomyopathies. ${ }^{1}$ Patients were medically treated with $\beta$-blockers, calcium channel blockers, antiarrhythmics and diuretics, as clinically indicated. Implantable cardioverter-defibrillators were utilised for primary or secondary prevention of sudden cardiac death.

Genetic testing assessed eight HCM-associated myofilament-encoding genes involving myosin-binding protein C (MYBPC3), $\beta$-myosin heavy chain (MYH7), essential and regulatory myosin light chains (MYL2, MYL3), cardiac troponin T (TNNT2), cardiac troponin I (TNNI3), $\alpha$-tropomyosin (TPM1) and cardiac actin (ACTC), as well as three genes associated with metabolic cardiomyopathies: $\alpha$-galactosidase (GLA) for Fabry's disease, LAMP2 for Danon's disease and PRKAG2 for PRKAG2 cardiomyopathy.

Written informed consent was obtained using a protocol approved by the Stanford Institutional Review Board. The investigation is in compliance with the principles outlined in the Declaration of Helsinki, ${ }^{4}$ state and national law and was approved by the Institutional Review Board at Stanford.

\section{Controls}

The control population included 100 prospectively recruited age-matched and gender-matched participants. A comprehensive health questionnaire was administered to these patients to exclude any symptoms suggestive of cardiovascular disease. We excluded subjects who had a family history of dilated cardiomyopathy or coronary artery disease in relatives younger than 55 years of age.

\section{Echocardiographic and Doppler studies}

Echocardiographic images were acquired on Philips IE 33 or HP 5500 (Andover, Massachusetts, USA) depending on the period of enrolment. Based on echocardiography, ventricular morphology was classified in various subgroups. Group 1: reverse curvature defined as a predominant mid-septal convexity towards the LV cavity, with the cavity itself having an overall crescent shape; group 2: hypertrophied with a proximal septal bulge (sigmoid); group 3: hypertrophied with a normal shape (symmetric); group 4: apical defined as a predominant apical distribution of hypertrophy (in the absence of significant hypertrophy among other LV segments); group 5: undefined if the morphology did not conform to one of the four defined subgroups and if an overall straight or variable convexity that was neither predominantly convex nor concave towards the LV cavity was present. ${ }^{5}$

Using M-mode and 2D, we measured the LV diameter, the thickness of the interventricular septum (IVS) and the LV posterior wall, as well as the left atrial (LA) endsystolic diameter according with the recommendations of the American Society of Echocardiography (ASE). ${ }^{6}$ The LVEF was assessed between the apical four-chamber and two-chamber view, using the biplane method of discs. ${ }^{7}$

The systolic intraventricular gradient was quantified using the continuous-Doppler technique. A peak gradient $>30 \mathrm{~mm} \mathrm{Hg}$ at rest was considered significant. ${ }^{8}$ Mitral regurgitation (MR) severity was assessed according to the ASE guidelines. ${ }^{9}$ LV filling was assessed by pulsed Doppler at the level of the mitral opening tips. The pattern of LV filling was classified as the restrictive filling pattern in the presence of E-deceleration time $<120 \mathrm{~ms}$ or of E/A wave $\geq 2$ associated with E-deceleration time $\leq 150 \mathrm{~ms}$, abnormal relaxation: E/A $<1$ associated with E-deceleration time $>220 \mathrm{~ms}$ or normal (or 'pseudonormal'): intermediate filling pattern. $^{10}$

We measured peak myocardial early diastolic velocity at the lateral mitral annulus (lateral $\mathrm{E}^{\prime}$ ) and transmitral to tissue Doppler imaging (TDI) early diastolic velocity ratio $\left(\mathrm{E} / \mathrm{E}^{\prime}\right.$; using tissue Doppler imaging). ${ }^{11}$

The LA and right atrial (RA) volumes were measured in systole just before the mitral valve opening, using a monoplane area-length method. ${ }^{12}$

According to the ASE guidelines, diastolic dysfunction was defined in the presence of severe LA dilation indexed left atrial LA volume $\left.(\mathrm{LAVi})>40 \mathrm{~mL} / \mathrm{m}^{2}\right)$, increased $\mathrm{E} / \mathrm{E}^{\prime}(>15)$, reduced $\mathrm{E}^{\prime}(<8 \mathrm{~cm} / \mathrm{s})$ and a restrictive pattern. ${ }^{13}$

Tricuspid annular plane systolic excursion (TAPSE) was measured from the systolic excursion of the tricuspid lateral annulus using a 2D methodology. According to the ASE guidelines, RV systolic dysfunction is considered in the presence of a TAPSE $<16 \mathrm{~mm}$ or a fractional area change (apical four-chamber view) $<35 \% .{ }^{14}$ The $\mathrm{RA}$ area was measured in systole just before the tricuspid valve opening.

\section{Statistical analysis}

Results are expressed as mean \pm SD for continuous variables or as the number of cases and percentage for 
Table 1 Main baseline characteristics of HCM population

Total $(n=383)$

\begin{tabular}{ll} 
Clinical and demographic & \\
Age (years) & $51 \pm 16$ \\
Male sex, n (\%) & $254(59)$ \\
NYHA III-IV, $\mathrm{n}(\%)$ & $56(15)$ \\
Echocardiography & \\
IVS (mm) & $17.7 \pm 5.1$ \\
PW (mm) & $12.1 \pm 3.1$ \\
RWT & $0.74 \pm 0.2$ \\
LVEF (\%) & $69 \pm 8$ \\
Resting LV gradient >30 mm Hg, n (\%) & $138(35)$ \\
Restrictive filling pattern, $\mathrm{n}(\%)$ & $14(4)$ \\
Moderate-to-severe MR, $\mathrm{n}(\%)$ & $24(6)$ \\
Medications & \\
Beta-blockers, $\mathrm{n}(\%)$ & $218(52)$ \\
Calcium channel blockers, $\mathrm{n}(\%)$ & $86(21)$ \\
ACE inhibitors, $\mathrm{n}(\%)$ & $65(16)$ \\
Diuretics, $\mathrm{n}(\%)$ & $50(13)$ \\
Antiarrhythmic therapy, $\mathrm{n}(\%)$ & $17(5)$ \\
ICD, $\mathrm{n}(\%)$ & $120(31)$ \\
\hline
\end{tabular}

ACE, angiotensin converting enzyme; EF, ejection fraction; $\mathrm{HCM}$, hypertrophic cardiomyopathy; ICD, implantable cardioverter defibrillator; IVS, interventricular septum; LAVi, left atrium volume index; LV, left ventricle; LVEF, left ventricle ejection fraction; MR, mitral regurgitation; PW, posterior wall; RAVi, right atrium volume index; RV, right ventricle; RVSP, right ventricle systolic pressure; RWT, relative wall thickness. categorical variables. Comparison of groups was performed using Student's t test for continuous variables with correction for unequal variance when necessary and $\chi^{2}$ test or Fisher's exact test, as appropriate for categorical variables. Comparisons between continuous variables among multiple groups were performed by analysis of variance-using the Brown-Forsythe statistic when the assumption of equal variances did not hold-while the proportions were compared by means of the $\chi^{2}$ test, using Fisher's exact test when necessary. To analyse the echocardiographic correlates of LV diastolic dysfunction, we considered the total population excluding the 16 patients with apical HCM (AHCM). Logistic regression analysis was used to determine the factors independently associated with LA dilation, reduced $\mathrm{E}^{\prime}$, increased $\mathrm{E} / \mathrm{E}^{\prime}$ and restrictive pattern. The first analysis considered the whole population while the second analysis included the subgroup of 166 patients with a genotype. To determine intrareader and inter-reader variability, the studies were read by two blinded investigators. A random sample of 25 studies was chosen to calculate the intrareader and inter-reader variability. Intrareader and interreader variability was quantified using mean differences as well as the intraclass correlation coefficient and no significant difference $(\mathrm{p}=0.81)$ between the two readers was observed. Statistical analysis was performed using the PASW software (PASW 18.0 Inc, Chicago, Illinois, USA).

Table 2 Characteristics of patient population in comparison with controls

\begin{tabular}{|c|c|c|c|}
\hline & Total HCM $(n=383)$ & Controls $(n=100)$ & p Value \\
\hline \multicolumn{4}{|l|}{ Clinical and demographic } \\
\hline Age (years) & $51 \pm 16$ & $52 \pm 16$ & NS \\
\hline Male sex, n (\%) & $227(59)$ & $62(62)$ & NS \\
\hline \multicolumn{4}{|l|}{ Echocardiography } \\
\hline IVS (mm) & $17.7 \pm 5.1$ & $7.4 \pm 1.2$ & $<0.001$ \\
\hline PW (mm) & $12.1 \pm 3.1$ & $7.4 \pm 1.1$ & $<0.001$ \\
\hline LVEF (\%) & $69 \pm 8$ & $65 \pm 6$ & NS \\
\hline E wave velocity $(\mathrm{cm} / \mathrm{s})$ & $83 \pm 27$ & $65 \pm 14$ & $<0.001$ \\
\hline A wave $(\mathrm{cm} / \mathrm{s})$ & $73 \pm 30$ & $57 \pm 20$ & $<0.001$ \\
\hline$E / A$ & $1.32 \pm 0.6$ & $1.27 \pm 0.5$ & NS \\
\hline Deceleration time (ms) & $210 \pm 63$ & $196 \pm 46$ & NS \\
\hline Restrictive filling pattern, n (\%) & $14(4)$ & $0(0)$ & 0.006 \\
\hline $\mathrm{E}^{\prime}$ & $8.14 \pm 3.39$ & $11.9 \pm 4.8$ & $<0.001$ \\
\hline$A^{\prime}$ & $7.71 \pm 3.0$ & $9.2 \pm 2.4$ & $<0.001$ \\
\hline$E / E^{\prime}$ & $12.31 \pm 7.91$ & $5.96 \pm 2.13$ & $<0.001$ \\
\hline LAVi $\left(m L / m^{2}\right)$ & $39.9 \pm 18.7$ & $26.3 \pm 19.1$ & $<0.001$ \\
\hline Moderate-to-severe MR, n (\%) & $24(6)$ & $0(0)$ & 0.01 \\
\hline Indexed RVEDA $\left(\mathrm{cm}^{2} / \mathrm{m}^{2}\right)$ & $8.8 \pm 2.4$ & $9.9 \pm 2.3$ & NS \\
\hline RVFAC (\%) & $49 \pm 8$ & $48 \pm 7$ & NS \\
\hline TAPSE<16 mm, n (\%) & $15(3)$ & $0(0)$ & $<0.001$ \\
\hline RV dysfunction*, n (\%) & $17(4)$ & $0(0)$ & $<0.001$ \\
\hline $\mathrm{RAVi}\left(\mathrm{mL} / \mathrm{m}^{2}\right)$ & $21.7 \pm 8.9$ & $21.6 \pm 9.4$ & NS \\
\hline RVSP mm Hg & $32 \pm 11$ & $19 \pm 7$ & $<0.001$ \\
\hline
\end{tabular}

*Defined as RVFAC $<33 \%$ and/or TAPSE $<16 \mathrm{~mm}$.

EF, ejection fraction; ICD, implantable cardioverter defibrillator; HCM, hypertrophic cardiomyopathy; IVS, interventricular septum; LAVi, left atrium volume index; LV, left ventricle; LVEF, left ventricle ejection fraction, MR, mitral regurgitation; PW, posterior wall; RAVi, right atrium volume index; RV, right ventricle; RVEDA, right ventricular end-diastolic area; RVFAC, right ventricular fractional area change; RVSP, right ventricle systolic pressure; TAPSE, tricuspid annular plane systolic excursion. 
Table 3 Characteristics of the patient population according to different morphology

\begin{tabular}{|c|c|c|c|c|c|c|}
\hline & $\begin{array}{l}\text { Group } 1 \\
(n=218)\end{array}$ & $\begin{array}{l}\text { Group } 2 \\
(n=27)\end{array}$ & $\begin{array}{l}\text { Group } 3 \\
(n=69)\end{array}$ & $\begin{array}{l}\text { Group } 4 \\
(n=16)\end{array}$ & $\begin{array}{l}\text { Group } 5 \\
(n=53)\end{array}$ & p Value \\
\hline \multicolumn{7}{|l|}{ Clinical and demographic } \\
\hline Age (years) & $50 \pm 17$ & $58 \pm 14$ & $49 \pm 18$ & $52 \pm 14$ & $51 \pm 14$ & 0.13 \\
\hline Male sex, n (\%) & $126(56)$ & $21(81)$ & $37(54)$ & $11(72)$ & $32(57)$ & 0.24 \\
\hline NYHA III-IV, n (\%) & $28(13)$ & $0(0)$ & $7(10)$ & $2(13)$ & $8(15)$ & 0.33 \\
\hline Genotype on 173 patients, $n$ (\%) & $53(55)$ & $5(38)$ & $12(39)$ & $2(29)$ & $12(48)$ & 0.37 \\
\hline \multicolumn{7}{|l|}{ Echocardiography } \\
\hline IVS (mm) & $19.7 \pm 5.2$ & $16.2 \pm 3.3$ & $14.9 \pm 4.1$ & $12.6 \pm 2.1$ & $15.4 \pm 3.4$ & $<0.001^{*}$ \\
\hline $\mathrm{PW}(\mathrm{mm})$ & $11.9 \pm 2.9$ & $11.4 \pm 2.0$ & $13.8 \pm 3.4$ & $10.7 \pm 2.5$ & $11.2 \pm 2.4$ & $<0.001^{*}$ \\
\hline LVEF (\%) & $70 \pm 7$ & $69 \pm 6$ & $68 \pm 9$ & $66 \pm 6$ & $69 \pm 9$ & 0.19 \\
\hline LV gradient $>30 \mathrm{~mm} \mathrm{Hg}, \mathrm{n}(\%)$ & $87(40)$ & $8(30)$ & $17(25)$ & $3(19)$ & $19(36)$ & $0.010 \dagger$ \\
\hline E wave $(\mathrm{cm} / \mathrm{s})$ & $82 \pm 26$ & $88 \pm 28$ & $85 \pm 27$ & $70 \pm 12$ & $91 \pm 31$ & $0.07 \dagger$ \\
\hline $\mathrm{E} / \mathrm{A}$ & $1.3 \pm 0.59$ & $1.1 \pm 0.46$ & $1.33 \pm 0.62$ & $1.5 \pm 0.55$ & $1.22 \pm 0.63$ & 0.19 \\
\hline$E / A>2, n(\%)$ & $21(10)$ & $1(4)$ & $9(15)$ & $3(19)$ & $3(6)$ & 0.34 \\
\hline Deceleration time (ms) & $212 \pm 62$ & $213 \pm 47$ & $207 \pm 77$ & $166 \pm 34$ & $213 \pm 64$ & 0.09 \\
\hline Restrictive pattern (n, \%) & $7(3)$ & $0(0)$ & $4(5)$ & $1(6)$ & $3(6)$ & $0.53 \ddagger$ \\
\hline$E^{\prime}(\mathrm{cm} / \mathrm{s})$ & $7.9 \pm 3.3$ & $8.1 \pm 2.6$ & $8.4 \pm 3.7$ & $8.7 \pm 3.5$ & $8.3 \pm 3.7$ & $0.82^{*}$ \\
\hline$E^{\prime}<12 \mathrm{~cm} / \mathrm{s}(\mathrm{n}, \%)$ & $160(86)$ & $23(88)$ & $44(79)$ & $12(86)$ & $39(81)$ & $0.65^{\star}$ \\
\hline$E / E^{\prime}$ & $12.3 \pm 7.9$ & $12.1 \pm 6.1$ & $12.7 \pm 9.5$ & $9.4 \pm 4.0$ & $12.7 \pm 7.9$ & $0.71^{*}$ \\
\hline LAVi mL/m² & $42.4 \pm 19.1$ & $36.2 \pm 10.5$ & $34.7 \pm 14.7$ & $38.6 \pm 13.6$ & $39.1 \pm 24.5$ & $0.03^{*}$ \\
\hline $\mathrm{LAVi}>40 \mathrm{~mL} / \mathrm{m}^{2}, \mathrm{n}(\%)$ & $102(47)$ & $9(33)$ & $22(32)$ & $6(37)$ & $17(32)$ & $0.09^{*}$ \\
\hline Moderate-to-severe MR & $15(7)$ & $2(7)$ & $3(4)$ & $1(6)$ & $3(6)$ & $0.96 \ddagger$ \\
\hline RV dysfunction, n (\%) & $11(5)$ & $0(0)$ & $3(0)$ & $0(0)$ & $3(6)$ & $0.19 \ddagger$ \\
\hline RVSP mm Hg & $33 \pm 11$ & $28 \pm 7$ & $30 \pm 9$ & $35 \pm 11$ & $30 \pm 9$ & $0.2^{*}$ \\
\hline $\operatorname{RAVi}\left(\mathrm{mL} / \mathrm{m}^{2}\right)$ & $21.7 \pm 8.9$ & $23.8 \pm 9.9$ & $20.4 \pm 9.3$ & $23.6 \pm 8.8$ & $22.1 \pm 7.6$ & 0.43 \\
\hline
\end{tabular}

Group 1: septal reverse; group 2: septal sigmoid; group 3: symmetric; group 4: apical; group 5: undefined.

$\mathrm{p}$ Value indicates the difference between subgroups according to morphology.

${ }^{*}$ All the morphology subgroups are significantly different $(p<0.05)$ in comparison with controls.

†Four morphology subgroups are significantly different $(p<0.05)$ in comparison with controls.

†Three morphology subgroups are significantly different $(p<0.05)$ in comparison with controls.

IVS, interventricular septum; LAVi, left atrium volume index; LV, left ventricle; LVEF, left ventricle ejection fraction, MR, mitral regurgitation;

$\mathrm{PW}$, posterior wall; RAVi, right atrium volume index; RV, right ventricle; RVSP, right ventricle systolic pressure.

\section{RESULTS}

\section{Patient characteristics}

The main baseline characteristics of the study population are summarised in table 1 . Mean age at the initial evaluation was $51 \pm 16$ years; 254 patients (59\%) were male, $56(15 \%)$ were symptomatic (NYHA III-IV) and $134(35 \%)$ had a significant rest LV gradient (either at the LVOT or mid-ventricular gradient). Eighty-four (48\%) patients had a known disease-related mutation, with a prevalence in MYH7 (n:40, 47\%). Patients were treated mainly with $\beta$-blockers (52\%) and calcium channel-blockers (21\%). A known ischaemic heart disease was present in $5 \%$ of cases.

\section{Diastolic parameters compared with the control population}

Diastole was impaired in the overall HCM population as compared with the healthy control subjects (table 2). Compared with controls, patients with HCM showed a greater left atrial size (LAVi; $39.9 \pm 18.7$ vs $26.3 \pm 19.1$, $\mathrm{p}<0.001)$, higher $\mathrm{E} / \mathrm{E}^{\prime}(12.31 \pm 7.91$ vs $5.96 \pm 2.13$, $\mathrm{p}<0.001)$ and significantly lower $\mathrm{E}^{\prime}$ values $(8.14 \pm 3.39$ vs $11.9 \pm 4.8$, $\mathrm{p}<0.001)$. A restrictive pattern was present in 14 patients with HCM (4\%), while it was absent in the control population.

\section{General features of patients divided by morphology}

Table 3 represents the clinical and echocardiographic measurements categorised by morphology. In total 218 $(57 \%)$ patients comprised group 1, while $27(7 \%), 69$ $(18 \%), 16(4 \%)$ and $53(14 \%)$ patients belonged to groups 2, 3, 4 and 5, respectively.

There was no significant difference in age at enrolment among the five groups, though patients with septal sigmoid morphology were slightly older $(50 \pm 17,58 \pm 14$, $49 \pm 18,52 \pm 14,50 \pm 14$ in groups $1,2,3,4$ and 5 , respectively, $\mathrm{p}=0.13$ ). Patients with reverse morphology were characterised by a more severe IVS hypertrophy (19.7 $\pm 5.2,16.2 \pm 3.3,14.9 \pm 4.1,12.6 \pm 2.1,15.4 \pm 3.4$ in groups 1 , 2, 3,4 and 5 , respectively, $\mathrm{p}<0.001)$.

\section{Diastolic parameters in subgroups with different morphology}

Diastole was similarly affected in each morphology subgroup (table 3). A restrictive pattern was rarely observed (3\% vs $0 \%$ vs $5 \%$ vs $6 \%$ vs $6 \%$ in groups $1,2,3,4$ and 5 , respectively, $\mathrm{p}=0.53)$. There was no difference in the $\mathrm{E}^{\prime}$ and $\mathrm{E} / \mathrm{E}^{\prime}$ ratio values between the HCM subgroups $\left(\mathrm{E}^{\prime}\right.$ $7.9 \pm 3.3$ in group $1,8.1 \pm 2.6$ in group $2,8.4 \pm 3.7$ in group $3,8.7 \pm 3.5$ in group $4,8.3 \pm 3.7$ in group $5, \mathrm{p}=0.82 ; \mathrm{E} / \mathrm{E}^{\prime}$ : 


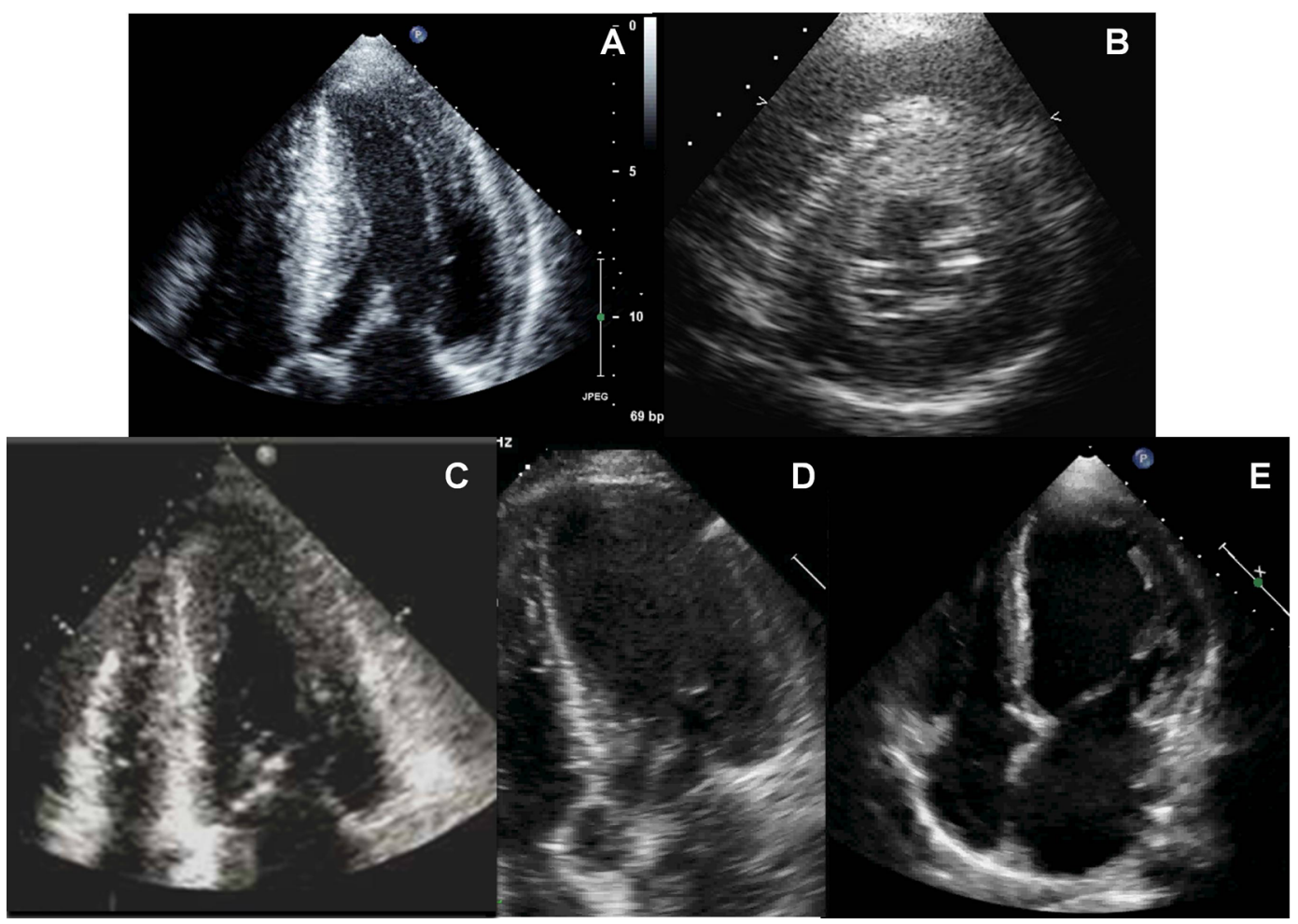

Figure 1 Two-dimensional images showing hypertrophic cardiomyopathy (HCM) morphological subtypes. Reverse curvature $(A)$; symmetric $(B)$; apical $(C)$; sigmoid $(D)$; indefinite, in this case end-stage HCM (E).

$12.3 \pm 7.9,12.1 \pm 6.1,12.7 \pm 9.5,9.4 \pm 4.0,12.7 \pm 7.9$ in groups $1,2,3,4$ and 5, respectively, $\mathrm{p}=0.71$; figure 1 ). Patients with reverse curvature morphology showed more severe LA dilation in comparison to patients with sigmoid morphology (LAVi 42.4 $\pm 19.1,36.2 \pm 10.5,34.7 \pm 14.7,38.6$ $\pm 13.6,39.1 \pm 24.5 \mathrm{~mL} / \mathrm{m}^{2}$ in groups $1,2,3,4$ and 5 , respectively, $\mathrm{p}=0.03)$. We compared the diastolic parameters of each HCM subgroup with those of the control population. $\mathrm{E}^{\prime}, \mathrm{E} / \mathrm{E}^{\prime}$ ratio and $\mathrm{LAVi}$ were different in each studied group compared with controls (figure 2).

\section{Right ventricular systolic pressure and relationship} with LV diastolic function

RVSP data were available on 222 patients (58\%). RVSP was higher in patients with $\mathrm{LAVi}>40 \mathrm{~mL} / \mathrm{m}^{2}(34 \pm 11$ vs 29 $\pm 9 \mathrm{mmHg}, \mathrm{p}=0.001)$, in patients with $\mathrm{E}^{\prime}<8(33 \pm 11$ vs 30 $\pm 9, \mathrm{p}=0.03)$ and in patients with $\mathrm{E} / \mathrm{E}^{\prime}>15(35 \pm 13$ vs $29 \pm 8$, $\mathrm{p}=0.003$; figure 3 ).

\section{Correlates of diastolic dysfunction}

Table 4 illustrates the independent correlates of diastolic dysfunction at multivariate analysis. LV gradient $>30 \mathrm{~mm} \mathrm{Hg}$ was associated with all three diastolic parameters considered (figure 4), in particular with $\mathrm{LAVi}>40 \mathrm{~mL} / \mathrm{m}^{2}$ (OR 2.04 (95\% CI 1.23 to 3.39$\left.), \mathrm{p}=0.005\right)$, $\mathrm{E} / \mathrm{E}^{\prime}>15$ (OR 4.66 (95\% CI 2.51 to 8.64$), \mathrm{p}<0.001$ ) and $\mathrm{E}^{\prime}$ $<8$ (OR 2.55 (95\% CI 1.42 to 4.53 ), $\mathrm{p}=0.001$ ). Also, age, moderate to severe MR, indexed IVS thickness and relative wall thickness (RWT) were associated with various diastolic parameters (table 4).

The only independent correlate of restrictive filling pattern was age (OR 0.94 (95\% CI 0.91 to 0.98 ), $\mathrm{p}=0.001$ ).

In order to analyse the possible contribution of the presence of one or more mutations, we also considered a model including the genotype (on 166 patients with data available). The presence of a disease-causing mutation was independently associated only with $\mathrm{E}^{\prime}<8$ (OR 2.52 (95\% CI 1.14 to 5.57$), \mathrm{p}=0.02)$.

\section{DISCUSSION}

Our study has two main findings. The first is that diastolic dysfunction equally affects patients with HCM even in the presence of a different distribution of hypertrophy. Each morphology subgroup compared with the age-matched and sex-matched control population was characterised by indicators of diastolic dysfunction of similar degree. The second is that diastolic dysfunction in HCM is associated with various clinical and echocardiographic variables as LV obstruction, age, degree of MR and LV hypertrophy. In particular, LV obstruction at rest was independently associated with all the echocardiographic indicators of diastolic dysfunction studied.

Diastolic dysfunction is a large contributor to the underlying pathophysiology in patients with HCM and it is a major characteristic of the disease. Its precise nature 

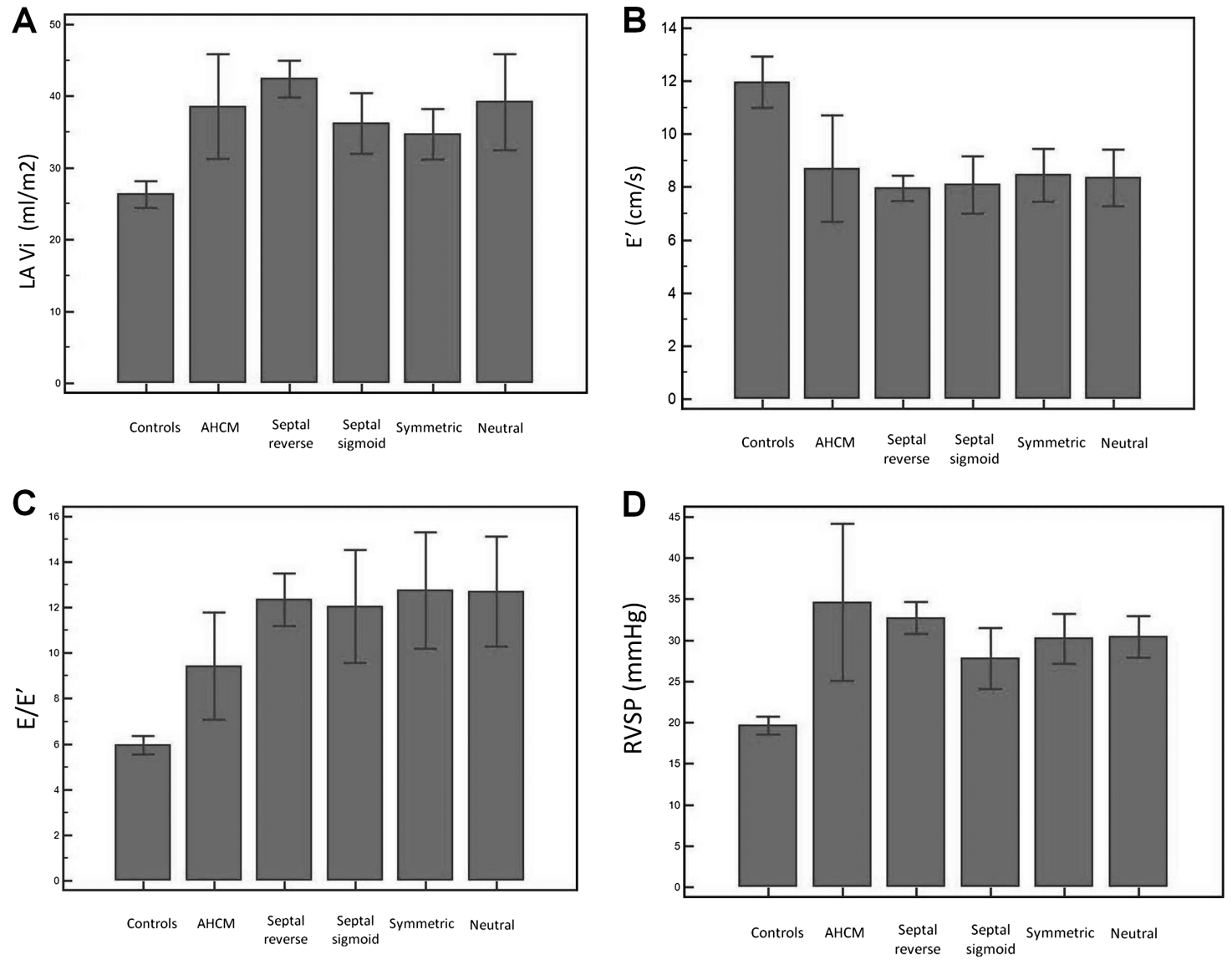

Figure 2 Diastolic dysfunction and RVSP in hypertrophic cardiomyopathy (HCM) population according to morphology and in controls. $p<0.001$ between subgroups in HCM and controls for all the four variable considered; $p=0.03$ for LAVi between septal reverse and symmetric; $p=N S$ for all the other variables in HCM subgroups. The values are expressed as means \pm 2 SDs. AHCM, apical hypertrophic cardiomyopathy; LAVi, left atrial indexed volume; RVSP, right ventricular systolic pressure.

and aetiology are complex and may be affected by several variables such as myocardial ischaemia, intracellular calcium overload, myocardial fibrosis and atrial haemodynamics. ${ }^{15}$ In several studies, some degree of impaired LV relaxation and reduced myocardial compliance has been present in the vast majority of patients with HCM and some studies have demonstrated that these factors are associated with poor prognosis. ${ }^{16-18}$ The importance of diastolic dysfunction in HCM has led to an extensive search for accurate, non-invasive methods of quantifying severity, but no single noninvasive measure has yet been validated. ${ }^{16}$

\section{Diastolic dysfunction and morphology in HCM}

The distribution of ventricular and septal wall thickening in HCM varies widely. Ventricular hypertrophy can be focal or diffuse, asymmetrical or concentric, obstructive or non-obstructive. HCM can be classified into at least five major anatomic subsets based on the septal contour, as well as the location and extent of hypertrophy: reverse curvature, sigmoidal, symmetric, apical and neutral contour. The most common HCM morphology is reverse curvature and it is most associated with identifiable HCM-associated gene mutations. ${ }^{5}$ One of the more unusual hypertrophic morphologies is AHCM. ${ }^{1}$ Although there are extensive investigations regarding diastolic function in patients with HCM, few studies have evaluated diastolic function according to the morphological pattern.

Ha et $a l^{19}$ studied a cohort of 29 patients with apical HCM. $\mathrm{E}^{\prime}$ was $<0.10 \mathrm{~m} / \mathrm{s}$ in all patients, while LA volume was above normal in $83 \%$ of the patients, suggesting the presence of chronic diastolic dysfunction. Elevated filling pressure (based in $\mathrm{E} / \mathrm{E}^{\prime}$ ) at rest was observed in approximately $20 \%$ of patients.

In our population, diastolic dysfunction was equally present in HCM characterised by different morphologies (comparing each group with a sex-matched control population). When myocardial relaxation is normal, $\mathrm{E}^{\prime}$ is usually $\geq 12 \mathrm{~cm} / \mathrm{s}$. In our study, $\mathrm{E}^{\prime}$ was $<12 \mathrm{~cm} / \mathrm{s}$ in the majority of patients, despite the presence of different morphologies, and $\mathrm{E}^{\prime}$ was significantly lower in each subgroup compared with the control population. Nagueh et $a l^{20}$ reported that when the mitral E velocity was corrected for the influence of myocardial relaxation (ie, the $\mathrm{E} / \mathrm{E}^{\prime}$ ratio), it correlated well with the mean pulmonary capillary wedge pressure. Geske $e t a l,{ }^{21}$ in a population of symptomatic patients with HCM, found 


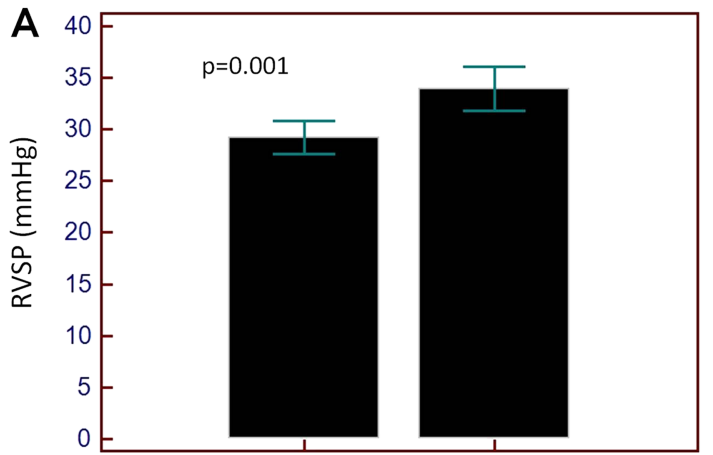

LAVi $\leq 40 \mathrm{ml} / \mathrm{m} 2 \quad$ LAVi $>40 \mathrm{ml} / \mathrm{m} 2$

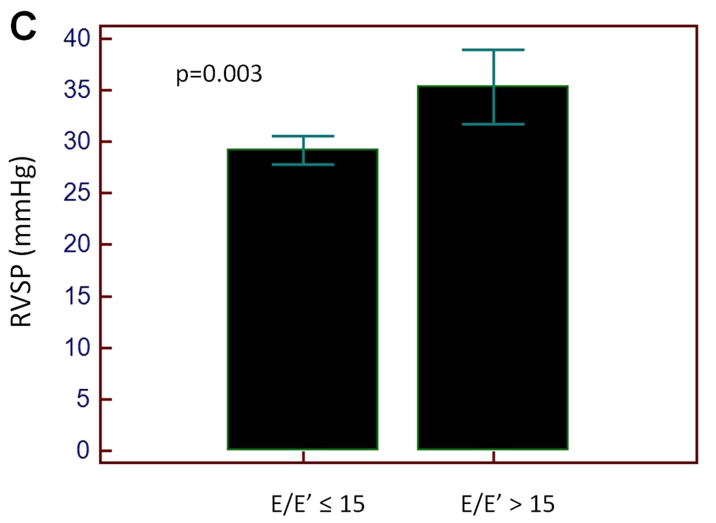

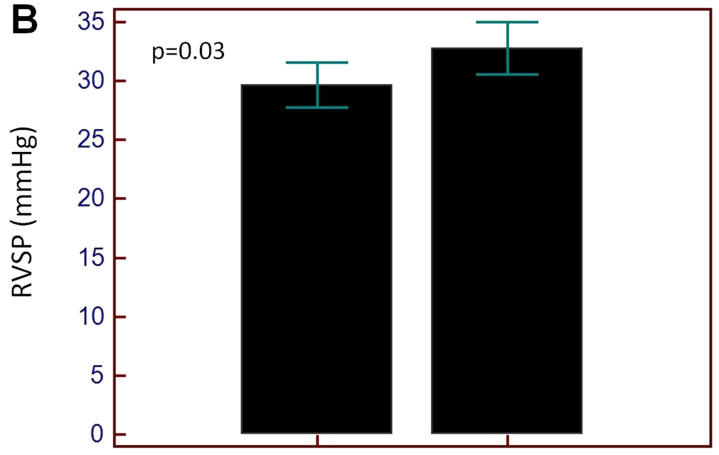

$E^{\prime} \geq 8 \mathrm{~cm} / \mathrm{s} \quad \mathrm{E}^{\prime}<8 \mathrm{~cm} / \mathrm{s}$

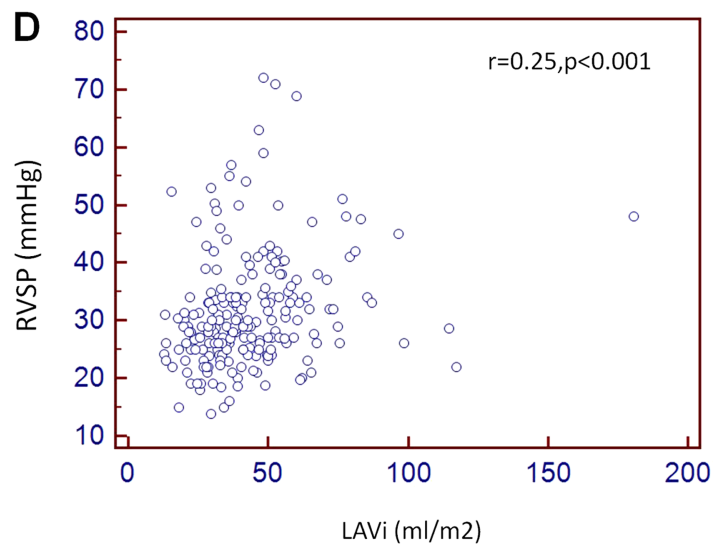

Figure 3 Right ventricular systolic pressure (RVSP) relationship with parameters of left ventricle (LV)diastolic dysfunction. The values are expressed as \pm 2 SDs. (A) RVSP and LAVi; (B) RVSP and E'; (C) RVSP and E/E'; (D) Correlation between LAVi and RVSP.

that Doppler echocardiographic estimates of LV filling pressure with the use of transmitral flows and mitral annular velocities correlate modestly with direct measurement of LAP at the catheterisation. In our cohort, the $\mathrm{E} / \mathrm{E}^{\prime}$ ratio was significantly elevated in each morphology subgroup.

LA volume, a reliable mirror of elevated LV and LA filling pressures in the absence of mitral valve disease, is considered to be an independent prognostic marker in patients with $\mathrm{HCM}^{22}$ In our population, a LA severe dilation (LAVi $>40 \mathrm{~mL} / \mathrm{m}^{2}$ ) was observed slightly more frequently in septal reverse form, but it was equally present in all the studied subgroups. It is striking that diastolic dysfunction was found to be not significantly affected by distribution of hypertrophy in the series studied. It could be hypothesised that morphologies different from reverse curvature forms (characterised by a more diffuse LV hypertrophy and by a very common presence of an identifiable HCM-associated mutation) may be affected by a less severe diastolic dysfunction. Our results argue against this hypothesis, underscoring that diastolic function appears to be independent from distribution of ventricular hypertrophy.

A parameter that did not emerge as significantly different between patients with HCM and controls was the $\mathrm{E} / \mathrm{A}$ ratio. This is probably related to the fact that patients with HCM can have a varied degree of diastolic dysfunction with, in some cases, a severe increase of the E/A ratio, but also an inverted diastolic transmitral Doppler inflow pattern. This result underscores the role of a multiparametric assessment of diastolic dysfunction.

\section{Correlates of diastolic dysfunction}

Diastolic dysfunction in HCM is complex and multifactorial, with changes at the molecular, myocardial tissue and global LV levels. ${ }^{23}$ Studies considering the correlates of diastolic dysfunction and focusing on echocardiographic parameters are lacking. It is difficult to establish if diastolic function in HCM is a main phenotypic expression, primarily related to a cellular and molecular mechanisms of abnormal relaxation, or it is more affected by a macroscopic subversion of the LV architecture, with a small LV cavity and, in some cases, an obstruction producing high intracavitary pressures. More than 400 mutations have been described in HCM, which result in the production of abnormal myocardial sarcomeric proteins producing an alteration of contraction and relaxation characteristics. ${ }^{24}$ These abnormalities vary with the sarcomeric protein affected and the site and effect of the mutation. ${ }^{25}$ Our study explored mainly the macroscopic part of the problem, but what emerged is that diastole is associated mainly with $\mathrm{LV}$ obstruction, then with age, degree of MR and LV hypertrophy. 

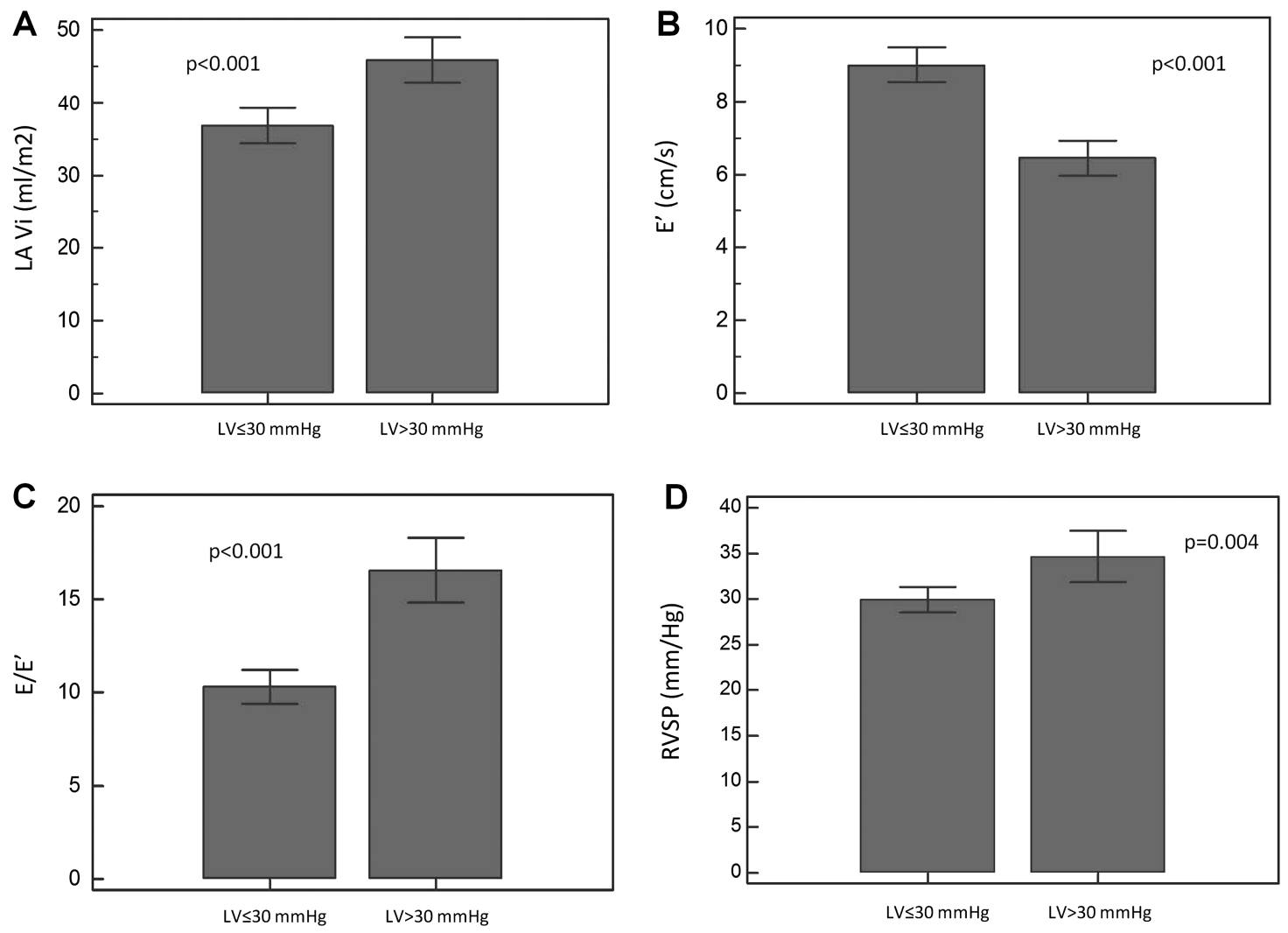

Figure 4 Presence of obstruction at rest and diastolic dysfunction. The values are expressed as means \pm 2 SDs. Left ventricle (LV) $>30 \mathrm{~mm}$ Hg: presence of LV obstruction at rest (LV gradient $>30 \mathrm{~mm} \mathrm{Hg}$ ). (A) LV>30 mmHg and LAVi; (B) LV>30 $\mathrm{mmHg}$ and $E^{\prime} ;(C) L V>30 \mathrm{mmHg}$ and $E / E^{\prime} ;(D) L V>30 \mathrm{mmHg}$ and RVSP.

The presence of at least one gene mutation appears to be independently related only to decreased $\mathrm{E}^{\prime}$, with no independent association with other parameters of diastolic dysfunction. Lower values of $\mathrm{E}^{\prime}$ at TDI have been previously demonstrated to predict the genetic status. ${ }^{26}$ Future studies are needed to better understand the distribution of genetic mutations according to different morphology. Our study explored simply the role of genetic status (mutation vs no mutation) in predicting diastolic abnormalities, but it is possible that certain disease-causing known mutations may have a direct role at a microscopic level that generates an overall diastolic

Table 4 Multivariate regression for diastolic dysfunction in all patients excluding apical HCM

\begin{tabular}{|c|c|c|c|c|c|c|}
\hline & \multicolumn{2}{|l|}{ LA volume $>40 \mathrm{~mL} / \mathrm{m}^{2}$} & \multicolumn{2}{|l|}{$E^{\prime}<8$} & \multicolumn{2}{|l|}{$E / E^{\prime}>15$} \\
\hline & OR (95\% Cl) & p Value & OR (95\% Cl) & p Value & OR (95\% Cl) & p Value \\
\hline \multicolumn{7}{|l|}{ Multivariate analysis } \\
\hline Age (years) & & & 1.05 (1.03 to 1.07$)$ & $<0.001$ & 1.04 (1.02 to 1.06$)$ & $<0.001$ \\
\hline \multicolumn{7}{|l|}{ Male sex } \\
\hline Indexed IVS (mm/m²) & 1.42 (1.24 to 1.62$)$ & $<0.001$ & & & $1.17(1.05-1.31)$ & 0.006 \\
\hline RWT (per 0.3 units) & & & 2.40 (1.58 to 3.64$)$ & $<0.001$ & & \\
\hline Moderate-to-severe MR & 6.10 (1.8 to 19.80$)$ & 0.003 & & & 6.38 (1.45 to 28.19$)$ & 0.01 \\
\hline $\begin{array}{l}\text { LV gradient }>30 \mathrm{~mm} \mathrm{Hg} \\
\text { Model adding genotype* }\end{array}$ & \multicolumn{4}{|c|}{ Model adding genotype* } & 4.66 (2.51 to 8.64$)$ & $<0.001$ \\
\hline Age (years) & & & 1.05 (1.02 to 1.08$)$ & $<0.001$ & 1.05 (1.01 to 1.08$)$ & 0.005 \\
\hline Male sex & & & 2.6 (1.65 to 3.82$)$ & 0.001 & $0.38(0.15$ to 0.96$)$ & 0.04 \\
\hline Indexed IVS $\left(\mathrm{mm} / \mathrm{m}^{2}\right)$ & 1.50 (1.95 to 1.89$)$ & $<0.001$ & & & & \\
\hline \multicolumn{7}{|l|}{ RWT (per 0.3 units) } \\
\hline Moderate-to-severe MR & $15.06(1.7$ to 130.55$)$ & 0.01 & & & 7.58 (1.89 to 30.2$)$ & 0.01 \\
\hline LV gradient $>30 \mathrm{~mm} \mathrm{Hg}$ & $2.17(1.01$ to 4.76$)$ & 0.05 & $2.89(1.26$ to 6.64$)$ & 0.01 & $6.73(2.67$ to 19.61$)$ & $<0.001$ \\
\hline Gene mutation $(\mathrm{s})^{*}$ & & & 2.52 (1.14 to 5.57$)$ & 0.02 & & \\
\hline
\end{tabular}

*On 166 patients.

IVS, interventricular septum; LV, left ventricle, MR, mitral regurgitation; RWT, relative wall thickness. 
dysfunction. Also, in recent years, several studies analysed with new sequencing methods the genome of patients affected by cardiomyopathies, and it is possible that in the next few years growing evidence will generate a better understanding of genotype/phenotype correlations in HCM.

At this point, we consider these variables as correlates; future multicentre studies are needed to validate these findings.

\section{Limitations}

The study has some limitations. The analysis did not include cardiac MR studies of the indexed mass (we also did not use Devereux' formula because it is inaccurate in patients with asymmetrical HCM) because these data were not available in the whole population. Thus, an evaluation of the possible relationship between diastolic function and cardiac mass was not possible. We did not consider pulmonary flow as a parameter to assess diastolic function, because it was available in a minority of patients.

The study was limited to clinical and echocardiographic data. Thus, invasive haemodynamics and biomarkers as brain natriuretic peptide (BNP) were available only in a minority of patients and a systematic comparison with cardiac catheterisation was not considered in the present study.

Generally, the variables considered to assess diastolic function (and, in particular, $\mathrm{E}$ and $\mathrm{A}$ wave velocity at the transmitral Doppler and $\mathrm{E} / \mathrm{E}^{\prime}$ ratio) are to a certain degree affected by loading conditions. Thus, $\mathrm{E}$ and $\mathrm{E}^{\prime}$ wave velocity may be higher in the presence of significant MR, due to an increased preload, and similarly, these values could be affected by the use of diuretics. It is thus in some way expected that significant MR is associated with $\mathrm{LA}$ enlargement and $\mathrm{E} / \mathrm{E}^{\prime}$ ratio. However, the presence of moderate-to-severe MR and the use of diuretics (in the majority of the cases thiazides rather than loop diuretics and at low dosages) were rare $(6 \%$ and $13 \%$, respectively). Besides, from our multivariate analysis, the other parameters considered (and, in particular, LV obstruction) were associated to diastolic dysfunction independently from MR.

The analysis is limited to the baseline; thus, prognostic implications and insights about the evolution of diastolic dysfunction during the follow-up cannot be inferred from our study.

\section{CONCLUSIONS}

Diastolic dysfunction, identified by various 2D and Doppler echocardiographic parameters, is prevalent in patients with HCM. Diastolic dysfunction does not appear to be strongly correlated with a particular morphological pattern despite the different distribution of LV hypertrophy. LV obstruction, age, degree of hypertrophy and MR are the main correlates of diastolic dysfunction. Future studies analysing genotype-phenotype associations may be able to clarify the various molecular mechanisms underlying diastolic dysfunction.

Contributors All the authors involved in the substantial contributions to conception and design, acquisition of data, or analysis and interpretation of data; drafting the article or revising it critically for important intellectual content; and final approval of the version to be published.

Funding Stanford Cardiovascular Institute and Biotronik Italia.

Competing interests EM is funded by the Sarnoff Cardiovascular Research Foundation.

Ethics approval Ethics approval was provided by the Institutional Review Board.

Provenance and peer review Not commissioned; externally peer reviewed.

Data sharing statement No additional data are available.

Open Access This is an Open Access article distributed in accordance with the Creative Commons Attribution Non Commercial (CC BY-NC 3.0) license, which permits others to distribute, remix, adapt, build upon this work noncommercially, and license their derivative works on different terms, provided the original work is properly cited and the use is non-commercial. See: http:// creativecommons.org/licenses/by-nc/3.0/

\section{REFERENCES}

1. Maron BJ, Maron MS. Hypertrophic cardiomyopathy. Lancet 2013;381:242-55.

2. Shah PM. Hypertrophic cardiomyopathy and diastolic dysfunction. J Am Coll Cardiol 2003;42:286-7.

3. Maron BJ, Maron MS, Wigle ED, et al. The 50-year history, controversy, and clinical implications of left ventricular outflow tract obstruction in hypertrophic cardiomyopathy from idiopathic hypertrophic subaortic stenosis to hypertrophic cardiomyopathy: from idiopathic hypertrophic subaortic stenosis to hypertrophic cardiomyopathy. J Am Coll Cardiol 2009;54:191-200.

4. Rickham PP. Human experimentation. Code of Ethics of the World Medical Association. Declaration of Helsinki. BMJ 1964;2:177.

5. Binder J, Ommen SR, Gersh BJ, et al. Echocardiography-guided genetic testing in hypertrophic cardiomyopathy: septal morphological features predict the presence of myofilament mutations. Mayo Clin Proc 2006;81:459-67.

6. Sahn DJ, DeMaria A, Kisslo J, et al. Recommendations regarding quantitation in M-mode echocardiography: results of a survey of echocardiographic measurements. Circulation 1978;58: 1072-83.

7. Lang RM, Bierig M, Devereux RB, et al. Recommendations for chamber quantification: a report from the American Society of Echocardiography's Guidelines and Standards Committee and the Chamber Quantification Writing Group, developed in conjunction with the European Association of Echocardiography, a branch of the European Society of Cardiology. J Am Soc Echocardiogr 2005;18:1440-63.

8. Sherrid MV, Wever-Pinzon O, Shah A, et al. Reflections of inflections in hypertrophic cardiomyopathy. J Am Coll Cardiol 2009;54:212-19.

9. Zoghbi WA, Enriquez-Sarano M, Foster E, et al. Recommendations for evaluation of the severity of native valvular regurgitation with two-dimensional and Doppler echocardiography. J Am Soc Echocardiogr 2003;16:777-802.

10. Nagueh SF. Echocardiographic assessment of left ventricular relaxation and cardiac filling pressures. Curr Heart Fail Rep 2009;6:154-9.

11. Yu CM, Sanderson JE, Marwick TH, et al. Tissue Doppler imaging a new prognosticator for cardiovascular diseases. J Am Coll Cardiol 2007;49:1903-14.

12. Le Tourneau T, Messika-Zeitoun D, Russo A, et al. Impact of left atrial volume on clinical outcome in organic mitral regurgitation. J Am Coll Cardiol 2010;56:570-8.

13. Nagueh SF, Appleton CP, Gillebert TC, et al. Recommendations for the evaluation of left ventricular diastolic function by echocardiography. J Am Soc Echocardiogr 2009;22:107-33.

14. Rudski LG, Lai WW, Afilalo J, et al. Guidelines for the echocardiographic assessment of the right heart in adults: a report from the American Society of Echocardiography endorsed by the European Association of Echocardiography, a registered branch of the European Society of Cardiology, and the Canadian Society of 
Echocardiography. J Am Soc Echocardiogr 2010;23:685-713; quiz 86-8.

15. Olivotto I, Cecchi F, Poggesi C, et al. Patterns of disease progression in hypertrophic cardiomyopathy: an individualized approach to clinical staging. Circ Heart Fail 2012;5: $535-46$.

16. Nagueh SF, Bierig SM, Budoff MJ, et al. American Society of Echocardiography clinical recommendations for multimodality cardiovascular imaging of patients with hypertrophic cardiomyopathy: endorsed by the American Society of Nuclear Cardiology, Society for Cardiovascular Magnetic Resonance, and Society of Cardiovascular Computed Tomography. J Am Soc Echocardiogr 2011;24:473-98.

17. Pinamonti $B$, Merlo M, Nangah R, et al. The progression of left ventricular systolic and diastolic dysfunctions in hypertrophic cardiomyopathy: clinical and prognostic significance. J Cardiovasc Med (Hagerstown) 2010;11:669-77.

18. Biagini E, Spirito P, Rocchi G, et al. Prognostic implications of the Doppler restrictive filling pattern in hypertrophic cardiomyopathy. Am J Cardiol 2009;104:1727-31.

19. Ha JW, Cho JR, Kim JM, et al. Tissue Doppler-derived indices predict exercise capacity in patients with apical hypertrophic cardiomyopathy. Chest 2005;128:3428-33.
20. Nagueh SF, Mikati I, Kopelen HA, et al. Doppler estimation of left ventricular filling pressure in sinus tachycardia. A new application of tissue Doppler imaging. Circulation 1998;98:1644-50.

21. Geske JB, Sorajja P, Nishimura RA, et al. Evaluation of left ventricular filling pressures by Doppler echocardiography in patients with hypertrophic cardiomyopathy: correlation with direct left atrial pressure measurement at cardiac catheterization. Circulation 2007;116:2702-8.

22. Nistri S, Olivotto I, Betocchi S, et al. Prognostic significance of left atrial size in patients with hypertrophic cardiomyopathy (from the Italian Registry for Hypertrophic Cardiomyopathy). Am J Cardiol 2006:98:960-5.

23. Ashrafian H, McKenna WJ, Watkins $\mathrm{H}$. Disease pathways and novel therapeutic targets in hypertrophic cardiomyopathy. Circ Res 2011;109:86-96.

24. Ho CY. Genetics and clinical destiny: improving care in hypertrophic cardiomyopathy. Circulation 2010;122:2430-40; discussion 40.

25. Teekakirikul P, Padera RF, Seidman JG, et al. Hypertrophic cardiomyopathy: translating cellular cross talk into therapeutics. J Cell Biol 2012;199:417-21.

26. Nagueh SF, McFalls J, Meyer D, et al. Tissue Doppler imaging predicts the development of hypertrophic cardiomyopathy in subjects with subclinical disease. Circulation 2003;108:395-8. 